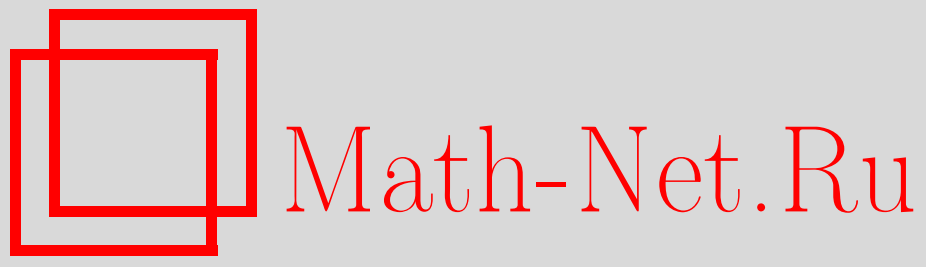

В. В. Горюнов, В. М. Закалюкин, Об устойчивости проекций лагранжевых многообразий с особенностями, Функи. анализ и его прил., 2004, том 38, выпуск 4, 13-21

DOI: https://doi.org/10.4213/faa123

Использование Общероссийского математического портала MathNet.Ru подразумевает, что вы прочитали и согласны с пользовательским соглашением

http://www.mathnet.ru/rus/agreement

Параметры загрузки:

IP: 3.93 .64 .190

26 апреля 2023 г., 12:37:00

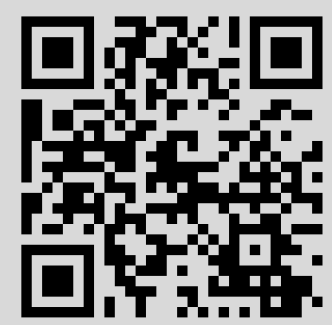


Функииональньй анализ и его приложения

2004, т. 38, вып. 4, с. 13-21

УДК 517.988

\title{
Об устойчивости проекций лагранжевых многообразий с особенностями*
}

\author{
(c) 2004. В. В. ГОрЮнов, В. М. ЗАКАЛЮКИН
}

Изучение лагранжевых и лежандровых подмногообразий с особенностями было начато В. И. Арнольдом примерно 25 лет назад при изучении особенностей в вариационной задаче обхода препятствия [1]. Первые примеры таких подмногообразий - раскрытые ласточкины хвосты - оказались связанными с дискриминантами некристаллографических групп Коксетера [4,8]. Разрабатывая общую теорию, включающую такие примеры, А. Б. Гивенталь [4] ввел понятие устойчивости лагранжева и лежандрова подмногообразий с особенностями по отношению к возмущениям только симплектической или контактной структуры и лагранжевой или соответственно лежандровой проекции при сохранении дифференцируемого типа подмногообразия.

Позже [7] было показано, что это понятие устойчивости имеет явное геометрическое описание в терминах производящих семейств, версальных деформаций особенностей функций и индуцирующих отображений.

В последнее время интерес к теории особых лагранжевых и лежандровых подмногообразий возрос в связи с их возможными приложениями к фробениусовым структурам, $D$-модулям и другим областям.

В этой работе мы переносим результаты работы [7] на случай некоторой естественной модификации понятия устойчивости по Гивенталю и показываем, что это условие устойчивости выполняется для широкого класса лагранжевых и лежандровых подмногообразий с особенностями, связанных с матричными особенностями (см. [2, 3,6]) и особенностями сложных функций [5].

\section{§1. 0-устойчивость}

В этом параграфе мы напоминаем некоторые основные понятия и вводим их модификации, которые будут использованы ниже.

1.1. Лагранжев случай. Особое лагранжево (под)многообразие $L$ симплектического пространства $M^{2 n}$ - это $n$-мерное аналитическое подмножество в $M$, являющееся лагранжевым в обычном смысле во всех своих регулярных точках. Лагранжева проекиия $\pi$ - это проекция $\pi: M \rightarrow B^{n}$, задающая расслоение, слои которого лагранжевы.

Слои всякого лагранжева расслоения имеют инвариантно определенную аффинную структуру. В самом деле, локальные координаты на базе поднимаются до регулярных функций на пространстве расслоения, которые попарно находятся в инволюции. Следовательно, отвечающие им гамильтоновы векторные поля не обращаются в нуль, коммутируют и касаются слоев.

* Работа второго автора выполнена при поддержке РФФИ (грант 02-01-00099) и программы «Университеты России» (грант UR0401016). 
Ограничение $\left.\pi\right|_{L}$ лагранжевой проекции $\pi$ на лагранжево подмногообразие $L \subset M$ называется лагранжевым отображением.

Два лагранжевых отображения лагранжевых подмногообразий $L^{\prime}$ и $L^{\prime \prime}$ называются эквивалентными, если существует симплектоморфизм соответствующих симплектических пространств, отображающий $L^{\prime}$ на $L^{\prime \prime}$, а слои одной лагранжевой проекции на слои другой. При этом, в частности, $L^{\prime}$ и $L^{\prime \prime}$ симплектоморфны.

Росток лагранжева отображения $\left.\pi\right|_{L}$ подмногообразия $L$ в точке $m$ называется устойчивым, если росток всякого лагранжева отображения $\left.\widetilde{\pi}\right|_{L}$, близкого к $\left.\pi\right|_{L}$, во всякой точке $\widetilde{m}$, близкой к $m$, эквивалентен ростку отображения $\left.\pi\right|_{L}$ в некоторой точке, близкой к $m$. Заметим, что в этом определение мы варьируем только расслоение $\pi$, оставляя подмногообразие $L$ фиксированным.

Согласно работе Гивенталя [4], это понятие устойчивости эквивалентно следующему понятию версальности ростка отображения $\left.\pi\right|_{L}$.

Пусть $\mathscr{O}_{L}$ - алгебра регулярных функций на $L$, и пусть $\mathbf{m}_{B, m}-$ максимальный идеал в алгебре $\mathscr{O}_{B, m}$ ростков функций на базе $B$ в точке $\pi(m)$. Определим локальную алгебру ростка отображения $\left.\pi\right|_{L}$ в точке $m$ как

$$
Q_{m}=\mathscr{O}_{L} /\left(\left(\left.\pi\right|_{L}\right)^{*}\left(\mathbf{m}_{B, m}\right)\right) \mathscr{O}_{L} .
$$

Алгебра $Q_{m}$ представляет собой алгебру ограничений функций, заданных на $L$, на пересечение $L$ со слоем $F_{\pi(m)}=\pi^{-1}(\pi(m))$.

Обозначим через $\mathscr{A}_{m}$ подпространство аффинных (по отношению к соответствующей аффинной структуре) функций на слое $F_{\pi(m)}$ и через $r: \mathscr{A}_{m} \rightarrow Q_{m}$ гомоморфизм ограничения, отправляющий функцию на слое в ее ограничение на $L \cap F_{\pi(m)}$.

Росток отображения $\left.\pi\right|_{L}$ в точке $m \in L$ называется версальным, если $r$ сюръективно, и миниверсальным, если $r$ - изоморфизм.

Пусть $p, q$ - локальные координаты Дарбу на $M$ в окрестности точки $m$ : $p(m)=p_{0}, q(m)=0$ и $\pi(p, q)=q$. По подготовительной теореме Вейерштрасса версальность ростка отображения $\left.\pi\right|_{L}$ в $m$ равносильна существованию для всякого аналитического ростка функции $\varphi$ на $M$ в точке $m=\left(p_{0}, 0\right)$ разложения следующего вида:

$$
\varphi(p, q)=\psi(p, q)+\sum_{j=1}^{n} a_{j}(q) p_{j}+a_{0}(q),
$$

где $a_{j}, j \geqslant 0,-$ аналитические ростки функций на базе $B$, а росток функции $\psi$ обращается в нуль на $L$.

ЗАмечАниЕ. Это разложение означает, что всякий росток в точке $m$ функции на $M$ является суммой функции, обращающейся в нуль на $L$, и функции, аффинной на каждом слое. Таким образом, всякое гамильтоново векторное поле в окрестности точки $m$ равно сумме гамильтонова поля, касающегося $L$, и гамильтонова поля, сохраняющего расслоение $\pi$. Применяя гомотопический метод, получим, что всякий росток симплектоморфизма пространства $M$ в точке $m$, близкий к тождественному, является композицией симплектоморфизма, сохраняющего $L$, и симплектоморфизма, сохраняющего стандартную проекцию $\pi$. Поскольку всякое возмущение ростка проекции $\pi$ в классе лагранжевых проекций является композицией проекции $\pi$ с подходящим симплектоморфизмом, из версальности вытекает устойчивость. Подробные доказательства см. в [4]. 
Обратимся теперь к специальному случаю этой теории. А именно, рассмотрим кокасательное расслоение $M=T^{*} B$ многообразия $B^{n}$ и отметим нулевое сечение $T_{0}$ в $T^{*} B$. Пусть $\operatorname{Sym}_{0}(M)$ - подгруппа симплектоморфизмов расслояния $M$, которые сохраняют $T_{0}$.

Два лагранжевых отображения лагранжевых подмногообразий кокасательного расслоения называются 0-эквивалентными, если они эквивалентны относительно симплектоморфизма из $\operatorname{Sym}_{0}(M)$.

Заменяя в определении устойчивости понятие эквивалентности на понятие 0-эквивалентности, получаем понятие 0-устойчивости ростка лагранжева отображения.

Нулевое сечение $T_{0}$ задает линейную структуру в слоях кокасательного расслоения. Заменяя пространство $\mathscr{A}_{m}$ аффинных функций на слое $F_{m}$ на инвариантно определенное подпространство $\mathscr{A}_{m}^{0}$ линейных функций, получаем определение 0-версальности, которое эквивалентно существованию для всякого ростка в точке $m$ функции $\varphi$ на $M$, такой, что $\left.\varphi\right|_{T_{0}}=0$, разложения вида

$$
\varphi(p, q)=\psi(p, q)+\sum_{j=1}^{n} a_{j}(q) p_{j}
$$

где ростки $a_{j}$ и $\psi$ такие же, как и в (1), причем мы предполагаем, что координаты Дарбу $p$ обращаются в нуль на нулевом сечении $T_{0}$.

Как и раньше, 0-версальность влечет за собой 0-устойчивость.

Кратность $\mu$ 0-миниверсального ростка лагранжева отображения, т. е. ранг локальной алгебры как векторного пространства, равна $n$. Она не превышает $n$, если росток 0-версален.

Мы ограничимся далее рассмотрением только комплексного случая. Отметим, что результаты этого раздела легко переносятся и в вещественную категорию.

ЛЕмма 1. Проекиия $\pi: T^{*} \mathbb{C}^{n} \rightarrow \mathbb{C}^{n},(p, q) \mapsto q$, лагранжева ростка $L$ в начале координат является 0-устойчивой, если и только если ростки произведений $p_{i} p_{j}, i, j=1, \ldots, n$, допускают разложсения

$$
p_{i} p_{j}=\psi_{i j}(p, q)+\sum_{k=1}^{n} c_{i j}^{k}(q) p_{k},
$$

в которых ростки функций $\psi_{i j}$ и $c_{i j}^{k}$ голоморфны и ростки $\psi_{i j}$ обращаются в нуль на L.

ДокАЗАтЕльство. Утверждение «только если» очевидно. Чтобы доказать «если», заметим, что идеал $I$, порожденный всеми квадратичными полиномами $P_{i j}(p)=p_{i} p_{j}-\sum c_{i j}^{k}(0) p_{k}, i, j=1, \ldots, n$, в пространстве голоморфных ростков функций на слое $F_{0}$ имеет конечную коразмерность. По модулю $I$ всякий росток функции на $F_{0}$ совпадает с ростком аффинной по $p$ функции. После проекции в локальную алгебру $Q_{0}$, т. е. после еще одной редукции по модулю функций, равных нулю на $L$ (точнее, на $L \cap F_{0}$ ), такая функция остается аффинной по $p$. Таким образом, условие 0-версальности выполняется.

Аналог этой леммы для устойчивости (а не 0-устойчивости) имеется в [4].

Надстройкой лагранжева расслоения $\pi: M \rightarrow B$ назовем его прямое произведение

$$
\widehat{\pi}=\left(\pi, \pi_{0}\right): \widehat{M}=M \times T^{*} \mathbb{C} \rightarrow B \times \mathbb{C}
$$


с канонической проекцией $\pi_{0}: T^{*} \mathbb{C} \rightarrow \mathbb{C}$.

Надстройкой лагранжева подмногообразия $L \subset M^{2 n}$ назовем $(n+1)$-мерное лагранжево подмногообразие $\widehat{L} \subset M \times T^{*} \mathbb{C}$, являющееся произведением подмногообразия $L$ и прямой $\ell=\left\{p_{n+1}=\right.$ const $\left.\neq 0\right\}$ в пространстве $T^{*} \mathbb{C}$, снабженным стандартными координатами Дарбу $p_{n+1}, q_{n+1}$.

Следующие утверждения непосредственно вытекают из определений.

ПреДЛОЖение 2. Росток отображсения $\left.\pi\right|_{L}$ в точке $m \in M$ (мини)версален тогда и только тогда, когда его надстройка $\left.\widehat{\pi}\right|_{\widehat{L}}$ 0-(мини)версальна в некоторой (и тогда - любой) точке линии $m \times \ell$ в пространстве $\widehat{M}$.

ПримеР. Росток стандартной проекции $\pi$ лагранжева подмногообразия $L \subset$ $T^{*} \mathbb{C}^{n}=\{p, q\}$, заданного производящим семейством $f=f(x, q)$ с параметрами $q \in \mathbb{C}^{n}$ и переменными $x \in \mathbb{C}^{k}$,

$$
L=\{(p, q) \mid \exists x: \partial f / \partial x=0, p=\partial f / \partial q\},
$$

является устойчивым тогда и только тогда, когда росток семейства $f(\cdot, \cdot)$ является $\mathscr{R}^{+}$-версальной деформацией ростка функции $f(\cdot, 0)$. Проекция является 0-устойчивой, если $f(\cdot, \cdot)$ является $\mathscr{R}$-версальной деформацией функции $f(\cdot, 0)$.

ПрЕДЛОЖЕНИЕ 3. Рассмотрим росток лагранжева подмногообразия $L$ в $\widehat{M}=T^{*} \mathbb{C}^{n} \times T^{*} \mathbb{C}$ в точке, не лежащей в нулевом сечении. Пусть росток отображсния $\left.\widehat{\pi}\right|_{L}$ является 0-версальным и L принадлежит регулярной гиперповерхности в пространстве $\widehat{M}$, трансверсалъной направлению $\partial / \partial p_{n+1}$. Тогда росток $\left.\widehat{\pi}\right|_{L}$ 0-эквивалентен надстройке версального ростка отображения $\left.\pi\right|_{L^{\prime}}$ некоторого лагранжева подмногообразия $L^{\prime} \subset M=T^{*} \mathbb{C}^{n}$.

1.2. Лежандров случай. Особое $n$-мерное подмногообразие контактного пространства называется лежсандровым, если во всех своих регулярных точках оно лежандрово в обычном смысле. Будем использовать стандартные (и эквивалентные) локальные модели контактных $(2 n+1)$-мерных пространств: проективизацию кокасательного расслоения $P T^{*} \mathbb{C}^{n+1}$ и пространство $J^{1}\left(\mathbb{C}^{n}, \mathbb{C}\right)=$ $\{p, q, z\}$ один-струй функций на $\mathbb{C}^{n}$, снабженное контактной формой $d z-p d q$. Определения лежандрова отображения, устойчивости и другие аналогичны лагранжеву случаю (см. также [4]).

Функторы симплектизации и контактизации связывают лагранжевы и лежандровы ростки следующим образом.

А. Проекция $\rho:(p, q, z) \mapsto(p, q)$ отображает лежандрово подмногообразие $\Lambda \subset J^{1}\left(\mathbb{C}^{n}, \mathbb{C}\right)$ на лагранжево подмногообразие $\rho(\Lambda) \subset T^{*} \mathbb{C}^{n}$.

В. Локально лагранжево расслоение и его нулевое сечение однозначно задают форму Лиувилля $\alpha=p d q$, являющуюся примитивной для симплектической формы $\omega=d \alpha$. Для заданного лагранжева ростка $L \subset T^{*} \mathbb{C}^{n}$ в точке $m$ обозначим через $L_{0, m}$ подмножество таких точек $s \in L$, что интеграл от $\alpha$ вдоль некоторого пути $\gamma$ на $L$, соединяющего $m$ и $s$, равен нулю.

Для простоты предположим, что значения интеграла не зависят от локального пути $\gamma$, т. е. что класс когомологий формы $\alpha$ на $L$ нулевой (примеры, когда такой класс ненулевой, см. в [4]).

Если $L_{0, m}$ не пересекает нулевое сечение расслоения $T^{*} \mathbb{C}^{n}$, то его проективизация является лежандровым (или изотропным) подмногообразием в $P T^{*} \mathbb{C}^{n}$. 
Его проекция $W_{0}(L, m)=\pi\left(L_{0, m}\right) \subset \mathbb{C}^{n}$ называется 0-волновым фронтом подмногообразия $L$.

С. Для лагранжева ростка $L \subset T^{*} \mathbb{C}^{n}$ в точке $m$ множество $\Lambda_{L, m} \subset J^{1}\left(\mathbb{C}^{n}, \mathbb{C}\right)$ таких точек $(p, q, z)$, что $s=(p, q) \in L$ и интеграл от формы $\alpha$ по пути в $L$, соединяющему $m$ и $s$, равен $z$, является лежандровым подмногообразием пространства струй $J^{1}\left(\mathbb{C}^{n}, \mathbb{C}\right)$.

Росток симплектоморфизма $\theta \in \operatorname{Sym}_{0}\left(T^{*} \mathbb{C}^{n}\right)$, сохраняющего проекцию $\pi$, сохраняет форму $\alpha$. Следовательно, если $\theta\left(L^{\prime}\right)=L^{\prime \prime}$, то $\theta\left(L_{0, m}^{\prime}\right)=L_{0, \theta(m)}^{\prime \prime}$. В координатах Дарбу диффеоморфизм $\theta$ имеет вид

$$
\theta:(p, q) \mapsto(P, \check{\theta}(q)),
$$

где $\check{\theta}-$ соответствующий диффеоморфизм базы и $P=\left(\check{\theta}^{-1}\right)^{*} p-$ значение на векторе $p$ линейного оператора на слое, сопряженного к оператору, обратному к производной диффеоморфизма $\theta$. В частности, $\check{\theta}\left(W_{0}\left(L^{\prime}, m\right)\right)=W_{0}\left(L^{\prime \prime}, \theta(m)\right)$.

Из этих определений вытекает следующее

ПРЕДЛОЖЕНИЕ 4. Рассмотрим лежандров росток $\Lambda \subset J^{1}\left(\mathbb{C}^{n}, \mathbb{C}\right)$ и предположим, что многообразие $\widehat{\rho(\Lambda)}$ не пересекает нулевое сечение и что его стандартная лагранжева проекиия 0-устойчива. Тогда проекиия ростка $\Lambda$ в $J^{0}\left(\mathbb{C}^{n}, \mathbb{C}\right)$ лежсандрово устойчива.

Обратно, если проекиия ростка $\Lambda$ в $J^{0}\left(\mathbb{C}^{n}, \mathbb{C}\right)$ лежсандрово устойчива и росток $\Lambda$ квазиоднороден при положстельных весах переменных, то росток $\widehat{\rho(\Lambda)}$ 0 -устойчив.

\section{§ 2. Устойчивость индуцированных отображений}

2.1. Теорема о критических значениях. Образы при лагранжевом отображении $\left.\pi\right|_{L}$ особых точек лагранжева подмногообразия $L$, а также образы критических точек сужения отображения $\left.\pi\right|_{L}$ на регулярную часть подмногообразия $L$ образуют каустику $\Sigma_{L}$ лагранжева отображения.

Каустика лагранжева ростка $L$ в точке $m$ конечной кратности $\mu$ является собственным аналитическим подмножеством базы $B$ положительной коразмерности.

Для точки $q \notin \Sigma_{L}$, близкой к отмеченной точке $\pi(m)$, прообраз $\pi^{-1}(q) \cap L$ состоит из $\mu$ различных точек $m_{i}$, близких к $m$. Можно считать, что локально $\pi$ является стандартным расслоением $T^{*} B \rightarrow B$. Это позволяет определить множество Максвелла $M_{L} \subset B$ как замыкание множества точек $q \notin \Sigma_{L}$, для которых $\mu$ значений переменной $z$ на $\Lambda_{L, m} \cap(\pi \circ \rho)^{-1}(q)$ не все различны. Если $\mu$ конечно, множество Максвелла задает росток собственного аналитического подмножества базы. Объединение каустики и множества Максвелла называется бифуркационной диаграммой $\operatorname{Bif}(\pi, L)$ лагранжевой проекции.

Рассмотрим лагранжеву проекцию $\pi: T^{*} \mathbb{C}^{n} \rightarrow \mathbb{C}^{n}$ ростка особого лагранжева подмногообразия $L$. Пусть $g: \mathbb{C}^{k} \rightarrow \mathbb{C}^{n}-$ росток гладкого отображения. При соответствующем выборе отмеченных точек ростков определим $u н \partial y$ цированное лагранжево отображение $g^{*}\left(\left.\pi\right|_{L}\right)$ как проекцию подмногообразия $g^{*}(L) \subset T^{*} \mathbb{C}^{k}$ на $\mathbb{C}^{k}$.

Tеорема 5. Пусть ростки проекиий $\left.\pi\right|_{L}$ в точке $m, m \notin T_{0}, u g^{*}\left(\left.\pi\right|_{L}\right)$ в точке $l$, такой, что $\rho(\pi(l))=\pi(m)$, являются соответственно 0-минивер- 
сальным и 0-устойчивым. Тогда множество критических значений $\Xi_{g}$ отображения $g$ содержится в объединении $W_{0}(L, m) \cup \operatorname{Bif}(\pi, L)$.

Заметим, что точка многообразия-прообраза называется критической, если производная отображения в ней не сюръективна. В частности, все точки прообраза критические, если его размерность меньше размерности образа. В этом случае из теоремы вытекает, что $g$ отображает $\mathbb{C}^{k}$ в $W_{0}(L, m) \cup \operatorname{Bif}(\pi, L)$.

Аналог этой теоремы для устойчивости доказан в [7].

ДокАЗАТЕЛЬСтво. Возьмем точку $q_{0} \in \mathbb{C}^{n} \backslash \Sigma_{L}$, близкую к отмеченной точ-

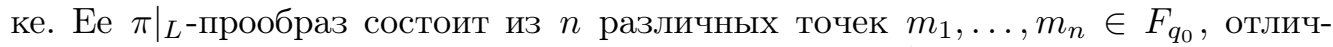
ных от начала координат. Мультиросток проекции $\left.\pi\right|_{L}$ в конечном множестве $\left\{m_{1}, \ldots, m_{n}\right\}$ является 0-версальным (разложение $(2)$ выполняется для мультиростков). Это равносильно тому, что точки $m_{i}$ линейно независимы в слое $F_{q_{0}}$ : ограничение произвольной функции на слое на это множество совпадает с ограничением некоторой линейной функции.

Пусть теперь $\lambda_{0} \in g^{-1}\left(q_{0}\right)$. Пусть $I \subset T_{q_{0}} \mathbb{C}^{n}$ - образ производной $g_{*}: T_{\lambda_{0}} \mathbb{C}^{k} \rightarrow$ $T_{q_{0}} \mathbb{C}^{n}$. Двойственное отображение $g^{*}: F_{q_{0}}^{n} \rightarrow F_{\lambda_{0}}^{k}$ между слоями кокасательных расслоений является композицией факторизации $p r$ слоя $F_{q_{0}}$ по подпространству $I^{\vee}$ ковекторов, аннулирующих $I$, и вложения. Предположим, что размерность $r$ подпространства $I^{\vee}$ положительна, т. е. что $\lambda_{0}-$ критическая точка отображения $g$. Из 0-устойчивости ростка $g^{*}\left(\left.\pi\right|_{L}\right)$ вытекает, что $p r$-образы линейно независимых точек $m_{1}, \ldots, m_{n} \in F_{q_{0}}$ образуют линейно независимый набор в $(n-r)$-мерном пространстве $F_{q_{0}} / I^{\vee}$ (здесь точки образа рассматриваются без учета кратности). Таким образом, набор вершин $\left\{m_{0}=0, m_{1}, \ldots, m_{n}\right\} n$ мерного симплекса $\sigma \subset F_{q_{0}}$ отображается в набор вершин $\left\{m_{0}^{\prime}=0, m_{1}^{\prime}, \ldots, m_{n-r}^{\prime}\right\}$ $(n-r)$-мерного симплекса в $F_{q_{0}} / I^{\vee}$. В частности, подпространство $I^{\vee}$ ранга $r$ порождается всеми такими разностями $m_{i}-m_{j}$, что $\operatorname{pr}\left(m_{i}\right)=\operatorname{pr}\left(m_{j}\right)$, т. е. векторами из всех граней $\sigma$, которые отображаются в точку при факторизации $p r$ (сумма размерностей таких граней равна $r$ ).

Вблизи каждой из точек $m_{i}, i=1, \ldots, n$, лагранжево многообразие $L$ является локально графиком дифференциала некоторой функции $z=\psi_{i}(q), \psi_{i}\left(q_{0}\right)=0$. Линейно независимые точки $m_{i} \in F_{q_{0}}$ являются дифференциалами функций $\psi_{i}$ в точке $q_{0}$.

Для всякой пары $i \neq j$ обозначим через $\Delta_{i j} \subset T_{q_{0}} \mathbb{C}^{n}$ гиперплоскость, касательную к гиперповерхности $\psi_{i}(q)-\psi_{j}(q)=0$.

Для каждого $\ell$ обозначим через $\Delta_{\ell} \subset T_{q_{0}} \mathbb{C}^{n}$ гиперплоскость, касательную к гиперповерхности $\psi_{\ell}(q)=0$. Гиперплоскости $\Delta_{\ell}$ и $\Delta_{i j}$ двойственны линиям направлений 1-мерных граней симплекса $\sigma \subset F_{q_{0}}$.

Условие того, что мультиросток $g^{*}\left(\left.\pi\right|_{L}\right)$ является 0-устойчивым в точках $F_{\lambda_{0}}$, равносильно тому, что подпространство $I$ совпадает с пересечением всех таких подпространств $\Delta_{\ell}$, что $\operatorname{pr}\left(m_{\ell}\right)=0$, и всех таких подпространств $\Delta_{i j}$, что $\operatorname{pr}\left(m_{i}\right)=\operatorname{pr}\left(m_{j}\right) \neq 0$. Таким образом, подпространство $I$ является пересечением подпространств в $T_{q_{0}} \mathbb{C}^{n}$, двойственных некоторым граням симплекса $\sigma$. Поскольку $I$ принадлежит касательному конусу в точке $q_{0}$ к множеству критических значений $\Xi_{g}$, регулярные страты множества $\Xi_{g}$ в окрестности $q_{0}$ совпадают с интегральными многообразиями распределений, заданных, подобно подпространству $I$, в касательных пространствах $T_{q} \mathbb{C}^{n}$ наборами граней соответствующих $n$-симплексов в слоях $F_{q}$. 
Из результатов статьи [7] (разд. 7.1 и 7.2) следует, что такие интегральные подмногообразия, имеющие максимальную размерность и содержащие точку $\pi(m)$ в своем замыкании, совпадают с регулярными стратами каустики, множества Максвелла и, как легко видеть, волнового фронта $W_{0}(L, m)$. Таким образом, $\Xi_{g} \subset W_{0}(L, m) \cup \operatorname{Bif}(\pi, L)$.

ТЕОРЕмА 6. Если $g$ является ростком собственного отображения пространств одинаковой размерности, то 0-устойчивость ростка $g^{*}\left(\left.\pi\right|_{L}\right)$ эквивалентна тому, что g является разветвленным накрытием, множество ветвления которого содержится в $W_{0}(L, m) \cup \operatorname{Bif}(\pi, L)$.

ДокАЗАтЕльство. В этом случае регулярные страты множества $\Xi_{g}$ являются $(n-1)$-мерными. По теореме 5 0-устойчивость влечет за собой требуемое свойство множества ветвления. Чтобы доказать обратное, достаточно заметить, что вне множества ветвления индуцированное отображение $g^{*}\left(\left.\pi\right|_{L}\right)$ является 0-миниверсальным.

Кроме того, оно является версальным и в регулярных точках множества ветвления, как легко видеть из действия на соответствующий симплекс в слое двойственного отображения $g^{*}$. Следовательно, всякий голоморфный росток функции $\varphi(p, q)$ допускает разложение $(2)$ с коэффициентами $a_{j}(q)$, однозначно определенными на дополнении к аналитическому подмножеству коразмерности, не меньшей 2. По теореме Хартогса такое разложение существует в целой окрестности отмеченной точки.

ЗАмЕчАНИЕ. Пусть росток лагранжева подмногообразия $L$ в точке $m \in T^{*} \mathbb{C}^{n}$ является надстройкой лагранжева ростка $L^{\prime}$ в точке, не принадлежащей нулевому сечению расслоения $T^{*} \mathbb{C}^{n-1}$. База $\mathbb{C}^{n}$ надстроенного лагранжева расслоения содержит выделенную координатную функцию $q_{n}$, соответствующую второму сомножителю произведения $L \simeq L^{\prime} \times \mathbb{C}$. Каустика и множество Максвелла для $L$ также являются произведениями каустики и множества Максвелла для $L^{\prime}$ на прямую (координатную ось $\left.q_{n}\right)$. С другой стороны, гиперплоскость, касательная к волновому фронту $W_{0}(L, m)$ в точке $m$, задана уравнением $d q_{n}=0$.

Если в условиях теоремы 6 множество ветвления $\Xi_{g}$ содержит $(n-1)$-мерную компоненту каустики или множества Максвелла, то направление $\partial_{q_{n}}$ принадлежит образу $I$ дифференциала отображения $g$ в точках, сколь угодно близких к $m$. Следовательно, сложная функция $q_{n} \circ g$ неособа в отмеченной точке. С другой стороны, если множество ветвления содержится в $(n-1)$-мерной компоненте волнового фронта $W_{0}(L, m)$, то отмеченная точка является критической для сложной функции $q_{n} \circ g$. В противном случае гиперплоскости, касательные к $\Xi_{g}$ вблизи отмеченной точки, не были бы близки к гиперплоскости $d q_{n}=0$.

2.2. Сложные функции. Интересный класс 0-устойчивых лагранжевых проекций доставляют версальные деформации составных отображений [6].

Для ростка функции $f:\left(\mathbb{C}^{n}, 0\right) \rightarrow(\mathbb{C}, 0)$ рассмотрим группу $\mathscr{K}_{f}$ (см. [6]), состоящую из ростков диффеоморфизмов $\Theta$ пространства-произведения $\mathbb{C}^{m} \times \mathbb{C}^{n}$ в точке $(0,0)$, расслоенных над проекцией на первый сомножитель, т. е. имеющих вид $\Theta:(x, y) \mapsto(X(x), Y(x, y)), x \in \mathbb{C}^{m}, y \in \mathbb{C}^{n}$, и таких, что $f(Y(x, y))=$ $f(y)$ при всех $(x, y)$.

Группа $\mathscr{K}_{f}$ естественно действует на пространстве ростков отображений $\varphi$ : $\left(\mathbb{C}^{m}, 0\right) \rightarrow\left(\mathbb{C}^{n}, 0\right)$, переводя график одного отображения в график другого. 
Пусть число Тюриной $\tau$ по отношению к группе $\mathscr{K}_{f}$ ростка отображения $\varphi$ в начале координат конечно. Пусть семейство $\Phi(x, \lambda)=\varphi(x)+\sum \lambda_{s} \varphi_{s}(x), \lambda \in \mathbb{C}^{\tau}$, является $\mathscr{K}_{f}$-миниверсальной деформацией отображения $\varphi$. Рассмотрим композицию $F=f \circ \Phi$.

Теорема 7. Лагранжева проекиия, заданная ростком производящего семейства $F(x, \lambda)$, является 0-устойчивой.

ДоказАтельство. Пусть $t \in(\mathbb{C}, 0)$ - дополнительный параметр. Рассмотрим деформацию

$$
F_{i j}=f \circ\left(\Phi+t \frac{\partial F}{\partial \lambda_{j}} \varphi_{i}\right)
$$

сложной функции $f \circ \varphi$. Поскольку $\left.F_{i j}\right|_{t=0}=F$ и $\Phi$ является $K_{f}$-версальной, то найдется семейство $K_{f}$-эквивалентостей, зависящее от $t$ и индуцирующее семейство $F_{i j}$ из семейства $F$ :

$$
F_{i j}(x, \lambda, t)=f \circ\left(\varphi(X(x, \lambda, t))+\sum_{s=1}^{\tau} \Lambda_{s}(\lambda, t) \varphi_{s}(X(x, \lambda, t))\right) .
$$

Более того, семейство эквивалентностей можно выбрать так, чтобы при $t=0$ отображение $(x, \lambda) \mapsto(X, \Lambda)$ было тождественным.

Дифференцируя предыдущее равенство по $t$ при $t=0$, получим

$$
\frac{\partial F}{\partial \lambda_{i}} \frac{\partial F}{\partial \lambda_{j}}=\sum \frac{\partial F}{\partial x_{r}} \frac{\partial X_{r}}{\partial t}+\sum \frac{\partial F}{\partial \lambda_{k}} \frac{\partial \Lambda_{k}}{\partial t} .
$$

Поскольку $\partial F / \partial \lambda_{i}=p_{i}$ и $\partial F / \partial x_{r}=0$ на лагранжевом подмногообразии, заданном производящим семейством $F$, это равенство совпадает с критерием 0 -устойчивости из леммы 1 для этого подмногообразия.

Пусть росток в нуле сложной функции $h=f \circ \varphi$ имеет конечную кратность $\mu$. Деформация $F=f \circ \Phi$ ростка $h$ индуцирована из $\mathscr{R}$-миниверсальной деформации $H$ ростка $h$ при помощи ростка отображения $g:\left(\mathbb{C}^{\tau}, 0\right) \rightarrow\left(\mathbb{C}^{\mu}, 0\right)$ пространств баз деформаций.

СлЕДСТвИЕ 8. Если $\tau=\mu$ и индуцирующее отображение $g$ собственное, то g является накрытием, разветвленным над 0-волновым фронтом 0-устойчивого лагранжева подмногообразия, заданного производящим семейством $H$.

ДокАЗАТЕЛЬСтво. Утверждение тривиально, если росток функции $f$ регулярен (в этом случае отображение $g$ является диффеоморфизмом). Предположим, что росток функции $f$ имеет в нуле критическую точку. В этом случае композиция отображения $g$ с проекцией $\mathbb{C}^{\mu} \rightarrow \mathbb{C}$ вдоль гиперплоскости, касательной к дискриминанту функции $h$ в нуле, особа в $0 \in \mathbb{C}^{\tau}$. Результат вытекает теперь из теорем 7,6 и замечания после теоремы 6.

ЗАмечАния. 1. Отображение накрытия, индуцирующее детерминантную функцию версальной матричной деформации простой матричной особенности из версальной деформации детерминантной функции невозмущенной матрицы (см. [6]), представляет собой частный случай отображения из следствия 8.

2. Пространство линейных функций на слое $F_{q}$ кокасательного расслоения $T^{*} B \rightarrow B$ совпадает с касательным пространством $T_{q} B$. Таким образом, функции $c_{i j}^{k}$, определенные разложением (3) для 0-версального лагранжева ростка, задают поточечное ассоциативное умножение ростков векторных полей на базе. 
3. В условиях следствия $8 \mathscr{K}_{f}$-дискриминант ростка $\varphi$ является свободным дивизором. Доказательство этого факта будет опубликовано отдельно.

\section{ЛитерАТУРА}

1. Арнолъд В. И. Критические точки функций на многообразии с краем, простые группы Ли $B_{k}, C_{k}, F_{4}$ и особенности эволют. УМН, 33, вып. 5, 91-105 (1978).

2. Bruce J. W. On families of symmetric matrices. Moscow Math. J., 3, No. 2, 335-360 (2003).

3. Bruce J. W., Goryunov V. V., Zakalyukin V. M. Sectional singularities and geometry of families of planar quadratic forms, Trends in Singularities, 83-97, Trends Math., Birkhäuser, Basel, 2002.

4. Гивенталь А. Б. Особые лагранжевы многообразия и их лагранжевы отображения. Итоги науки и техники, Современные проблемы математики. Новейшие достижения, т. 33, ВИНИТИ, М., 1988, с. 55-112.

5. Goryunov V. V., Mond D. Tjurina and Milnor numbers of matrix singularities. arXiv:math.AG/0307025.

6. Goryunov V. V., Zakalyukin V. M. Simple symmetric matrix singularities and the subgroups of Weyl groups $A_{\mu}, D_{\mu}, E_{\mu}$. Moscow Math. J., 3, No. 2, 507-530 (2003).

7. Робертс P. М., Закалюкин B. M. Устойчивость лагранжевых многообразий с особенностями. Функц. анализ и его прил., 26, вып. 3, 28-34 (1992).

8. Щербак О. П. Волновые фронты и группы отражений. УМН, 43, вып. 3, 125-160 (1988).

Университет Ливерпуля, Великобритания email: Victor.Goryunov@liverpool.ac.uk 